\title{
EVALUACIÓN DEL ESTADO DE CONSERVACIÓN DE CUATRO ESPECIES DE AVES DE LA FAMILIA RHINOCRYPTIDAE EN UN ÁREA SILVESTRE PROTEGIDA EN EL SUR DE CHILE
}

\author{
Yenny SOTO-MORA ${ }^{1}$ y Jonathan URRUTIA ${ }^{2}$ \\ 1Escuela de Ciencias Ambientales, Facultad de Recursos Naturales, Universidad Católica de Temuco, \\ Casilla 15-D, Temuco, CHILE. yennysotomora@gmail.com \\ ${ }^{2}$ Escuela de Ciencias Ambientales, Facultad de Recursos Naturales, Universidad Católica de Temuco, \\ Casilla 15-D, Temuco, CHILE. jurrutiaestrada@gmail.com
}

Soto-Mora, Y. \& Urrutia, J. 2010. Evaluación del Estado de Conservación de cuatro especies de aves de la Familia Rhinocryptidae en un Área Silvestre Protegida en el Sur de Chile. Acta Zool. Mex. (n.s.), 26(2): 401-414.

RESUMEN. La riqueza de avifauna endémica de Chile está siendo amenazada por procesos de destrucción de sus hábitats naturales. Debido a ello se evalúa el estado de conservación de cuatro especies pertenecientes a la Familia Rhinocryptidae, característica de los bosques templados de Sudamérica, presentes en un área silvestre protegida. Para ello se utilizó el Método propuesto por Reca et al. (1994), quienes emplean el índice SUMIN, compuesto por valores de 12 variables. Los resultados obtenidos señalan que Eugralla paradoxa (churrín de la Mocha) presenta Prioridad Máxima (PM) de ser conservada, Scelorchilus rubecula (chucao) merece una Atención Especial (AE) de conservación, en cambio Pteroptochos tarnii (hued hued del sur) y Scytalopus magellanicus (churrín del sur) serían especies No Prioritarias de conservar (NP). De la aplicación de este método, se deduce que es necesario implementar medidas de protección concretas para esta familia, ya que su hábitat presenta indicios de intervención antrópica, lo que se puede traducir en una disminución de sus poblaciones.

Palabras clave: Índice SUMIN, Método Reca, Rhinocryptidae.

Soto-Mora, Y. \& Urrutia, J. 2010. Assessment of the conservation status of four bird species of the Rhinocryptidae family in a protected forest area in Southern Chile. Acta Zool. Mex. (n.s.), 26(2): 401-414.

ABSTRACT. In Chile, the richness of endemic birds has been threatened by destruction processes of their natural habitats. Due to this being evaluated the conservation status of four species belonging to the Rhinocryptidae. This family is characteristic of the temperate forests of South America, an it is in a protected forest area. The evaluation was made using the Method proposed by Reca et al. (1994), which use the SUMIN index consisting of 12 variables. The results indicate that Eugralla paradoxa (ochre-flanked tapaculo) presents Maximum Priority (PM) to be preserved, Scelorchilus rubecula (chucao tapaculo) deserves Special Attention (AE) of conservation, while Pteroptochos tarnii (black throated huet huet) and Scytalopus magellanicus (andean tapaculo) species would Not preserve Priority (NP). After this, it is necessary to implement a specific protective measurements for this

Recibido: 08/10/2009; aceptado: 23/03/2010. 
family, given that its habitat shows signs of antropogenic processes, which may translate into a declining of their populations.

Key Words: SUMIN index, Method Reca, Rhinocryptidae.

\section{INTRODUCCIÓN}

La Familia Rhinocryptidae a nivel mundial está constituida por 12 géneros y más de 35 especies (Fjeldsâ \& Krabbe 1990), presentándose una especie en estado Vulnerable, tres especies en Peligro de Extinción y dos en Peligro Crítico de Extinción (UICN 2007). El alto nivel de endemismo y la baja relación especie/género presente en la Familia evidencian el carácter relictual y único. Su origen relicto del cenozoico temprano, con taxa originados en austrosudamérica explican su presencia desde América Central (Vuilleumier 1985), extendiéndose por el cordón andino (Fjeldsâ \& Krabbe 1990), desde Costa Rica al Cabo de Hornos (UICN 2007), constituyéndose en una de las familias de aves más primitivas en los bosques templados de Sudamérica (Feduccia \& Olson 1982), además de ser considerada como bioindicadora del grado de naturalidad del bosque y de ambientes prístinos, es decir, especie biológica que define un rasgo o característica del medio ambiente, así como también de su importancia como especies paraguas, puesto que cubren grandes áreas en sus desplazamientos diarios o estacionales, esto sugiere que conservando un hábitat suficiente como para asegurar una población viable de estas especies, entonces se benefician otros organismos de áreas más restringidas (Sarasola et al. 1999).

En Chile la Familia Rhinocryptidae habita en forma permanente en bosques templados lluviosos del sur de Chile (Rozzi et al. 1996, Sieving et al. 2000), aunque se ha registrado su presencia desde Atacama hasta Tierra del Fuego (Araya et al. 1996). A la fecha se han registrado ocho especies y tres subespecies, todas endémicas a esta región (Araya et al. 1996, Rozzi et al. 1996), a excepción de Scytalopus magellanicus (Gmelin, 1789) originario de Sudamérica austral (Rozzi et al. 1996). En los Libros Rojos Chilenos (Glade 1993, SAG 1998, 2007) no se han incluido el estado de conservación de las especies de rinocríptidos chilenos y por tanto, no se ha documentado el estado actual de conservación y/o amenaza de ellos. Dada la significativa reducción y/o alteración del hábitat para estas especies en Chile. El objetivo del presente estudio ha sido evaluar el estado de conservación y/o amenaza de los rinocríptidos chilenos a través de la Metodología propuesta por Reca et al. (1994).

\section{Área de estudio}

\section{MATERIAL Y MÉTODOS}

El Monumento Natural Cerro Nielol (MNCÑ, 89 ha), integrante de la SNASPE de la región de la Araucanía, se localiza en el valle central de la Región, Temuco, Chile ( $38^{\circ} 43^{\prime} \mathrm{S}$ y $72^{\circ} 35^{\prime} \mathrm{W}$ ), entre los 115 y $322 \mathrm{~m}$ de altitud (INE 1988). Se caracteriza por presentar áreas de vegetación nativa $(57 \%)$ y otras con vegetación introducida 
(43\%) (Hauenstein et al. 1988). Posee la Formación Vegetacional "Bosque Caducifolio del Llano", albergando a uno de los últimos bosques nativos de la depresión intermedia del valle centro-sur de Chile (Gajardo 1983), presentando especies representativas de tres unidades vegetacionales (bosque higrófilo templado, bosque esclerófilo y bosque semi-esclerófilo o semi-deciduo) (Hauenstein et al. 1988), en la que la formación vegetacional predominante corresponde al bosque parcialmente caducifolio de Roble-Laurel-Lingue (Nothofago-Perseetum linguae) (Tomaselli 1981). El MNCÑ al estar rodeado de centros poblacionales y zonas de labranza, el Monumento se ha convertido en una verdadera isla vegetacional, que acoge a un sinnúmero de especies. Por otro lado, las influencias climáticas cálidas que recibe de la zona central, unida a la humedad de la selva valdiviana, lo convierten en un área de gran diversidad biológica, presentando un clima del tipo mediterráneo perhúmedo (Csb3) (Di Castri \& Hajek 1976), de verano seco con una corta estación de sequía (Köeppen 1931), con temperatura promedio de $12^{\circ} \mathrm{C}$, humedad relativa de $80 \%$ y una precipitación media anual de $1324 \mathrm{~mm}$ (Di Castri \& Hajek 1976). El área de estudio forma parte de la formación volcánica efusiva-extrusiva denominada Cordón Huimpil-Ñielol, de suelos rojos arcillosos, relativamente básicos, con un alto porcentaje de materia orgánica (CIREN 2002). En cuanto a su hidrografía, no existen cursos de agua regulares, salvo pequeñas vertientes naturales de escaso caudal.

\section{Selección de las áreas de muestreo}

Se realizó una prospección total del área de estudio a fin de establecer los puntos de muestreo, procediendo a censar durante las estaciones de invierno, primavera, verano y otoño de 2007-2008. Los criterios para la selección fueron: lugares con mayores registros de rinocríptidos, accesibilidad, sectores prístinos (sin intervención turística) y con intervención turística, presencia de vegetación arbórea nativa e introducida, y presencia de vertientes naturales de agua. Con el fin de estimar la abundancia local, el muestro de aves se realizó en nueve estaciones, dos ubicadas en el sendero Agua Santa, tres en sendero Los Copihues, una en sector acceso antenas y tres en sendero Los Lingues. En cada época del año (invierno, primavera, verano y otoño), las zonas fueron censadas cinco veces cada una, durante las primeras horas de la mañana, a contar de las 07:00 a 10:30 h, mediante puntos de observación y de escucha de 5-10 min (Bibby et al. 1993), con radios de amplitud variable (60-100 m) cuidando que los puntos no se traslapen, ya que los puntos de conteo con radio fijo no son muy satisfactorios, al subestimar el número de especies observadas (Bibby et al. 1993). Para determinar las abundancias se procedió a censar la avifauna acompañante utilizando la misma metodología para los rinocríptidos (Bibby et al. 1993), teniendo la abundancia total (número total de individuos de todas las especies) y la relativa (proporción relativa de las diferentes especies en la comunidad) (Krebs 1986), la cual se expresa en la siguiente fórmula: $n_{i} / N$ X $100 \%=A B \%$; donde $n_{i}=$ Número total de 
individuos por especie, vistos o escuchados; $\mathrm{N}=$ Número total de individuos de todas las especies; $\mathrm{AB} \%=\mathrm{Abundancia}$ relativa.

\section{Estados de conservación}

Para la determinación del estado de conservación/ amenaza de las especies de rinocríptidos en el lugar de muestreo, se utilizó el método propuesto por Reca et al. (1994). Este método establece un índice de prioridades de conservación (SUMIN) basado en 12 variables: Distribución Continental (DICON), Distribución Nacional (DINAC), Amplitud en el Uso del Hábitat (AUHA), Amplitud en el Uso del Espacio Vertical (AUEVE), Tamaño Corporal (TAM), Potencial Reproductivo (POTRE), Amplitud Trófica (AMTRO), Abundancia Local (ABLOC), Singularidad Taxonómica (SINTA) (referido sólo a la presencia de taxón monotípico), Singularidad (SING) (especies con características únicas y/o propias), Acciones Extractivas (ACEXT) y Grado de Protección de las Especies (PROT)) (Grigera et al. 1996) (Apéndice 1).

Para cada especie a evaluar, se tuvo que asignar a las variables un valor numérico dentro de un rango determinado (0-5), correspondiendo el valor más alto a la situación más adversa para la especie, mientras que los valores cercanos a cero se presentan como lo más beneficioso para la especie. El valor de SUMIN, pertenece a la suma de las ponderaciones de cada variable, pudiendo tomar valores finales entre 0 y 30 , lo que significa que la mayor valoración (30) correspondería a la especie más susceptible o de mayor prioridad de ser conservada. (Apéndice 1). Posteriormente se calcula el valor medio del índice para el conjunto evaluado y si bien no se consignan explícitamente categorías, se determinan tres grupos de especies: "No Prioritarias", aquellas cuyo índice es menor que la media menos un valor estándar; "Atención Especial", si tienen un índice igual o mayor que la media; y "Prioridad Máxima", cuando el índice es igual o mayor que la media más un valor estándar. Todas las variables fueron obtenidas a partir de revisiones bibliográficas, a excepción de la Abundancia Local (ABLOC), que se estimó mediante trabajo de campo.

Para obtener la variable Abundancia Local (ABLOC) de las especies de aves se tomó como base los datos anuales del número de individuos por especie $\left(\mathrm{n}_{\mathrm{i}}\right)$ y el número total de individuos $(\mathrm{N})$ derivados del muestreo en terreno para obtener un porcentaje de abundancia (AB\%, Krebs 1986). Luego se obtuvo la media y desviación estándar de los resultados de porcentajes de abundancia; donde posteriormente, fueron categorizadas las abundancias locales, siendo "abundante o común" la especie que presente valores superiores a la sumatoria de la media más la desviación estándar, mientras que se considera "Escasa" a la especie que presenta un valor entre la media y la desviación estándar, siendo "Rara o muy rara" la especie con una abundancia inferior a los valores obtenidos de la media (Grigera et al. 1996). 


\section{RESULTADOS}

En la Apéndice II, se presentan los resultados de la valoración de cada una de las variables según método de Reca et al. (1994) para las cuatro especies de la Familia Rhinocryptidae presente en el MNCÑ. En el Apéndice 2 se indican los valores del SUMIN obtenido y la categoría asignada a cada especie. Los valores sugieren a dos especies en peligro, el churrín de la Mocha (Eugralla paradoxa) con un SUMIN de 19. Prioridad Máxima; y el chucao (Scelorchilus rubecula) un SUMIN de 18, Atención Especial (AE). Las dos especies restantes, Hued-hued del sur (Pteroptochos tarnii) y churrín del sur (Scytalopus magellanicus), fueron categorizadas como No Prioritarias (NP) (Cuadro 1) para su conservación, con un SUMIN de 17 y 15 respectivamente.

Cuadro 1. Categorías de conservación de miembros de la Familia Rhinocryptidae presentes en el MN Cerro Nielol. (RECA et al. (1994)): PM= Prioridad Máxima, AE= Atención Especial, $\mathrm{NP}=$ No Prioritarias, SAG (1998): B= Especie catalogada como benéfica para la actividad silvoagropecuaria, UICN (2004, 2007): LC= Preocupación Mínima).

\begin{tabular}{lccccc}
\hline Especie & $\begin{array}{c}\text { Glade } \\
(\mathbf{1 9 9 3})\end{array}$ & $\begin{array}{c}\text { RECA } \text { et al. } \\
\mathbf{( 1 9 9 4 )}\end{array}$ & $\begin{array}{c}\text { SAG } \\
(\mathbf{1 9 9 8})\end{array}$ & $\begin{array}{c}\text { UICN } \\
(\mathbf{2 0 0 4}, \mathbf{2 0 0 7})\end{array}$ \\
\hline P. tarnii & - & NPt.3 & Art.4 & \\
S. rubecula & - & AE & B & - & LC \\
E. paradoxa & - & PM & B & - & LC \\
S. magellanicus & - & NP & B & - & LC \\
\hline
\end{tabular}

\section{DISCUSIÓN}

La Familia Rhinocryptidae, presenta hoy en día muchos impactos derivados de la intervención antrópica, sin embargo, uno de los más importantes es el proceso de fragmentación que está viviendo el MNCÑ, hábitat de estas especies sensibles e indicadoras del grado de pristinidad de los bosques (Sarasola et al. 1999), ya que representan a especies sensitivas dentro de una región y en general actúan como señal de alarma para alertar a los biólogos que monitorean las condiciones ambientales. Los rinocríptidos son especies que no tienen una capacidad de dispersión efectiva en relación a sus desplazamientos, debido a que vuelan muy poco $(<50 \mathrm{~m})$ (Rozzi et al. 1996), presentando alas cortas, lo que restringe su movilidad en hábitats fragmentados por lo que estarían vulnerables ante los efectos antrópicos sobre el bosque. Esta familia es más sensible a la fragmentación (Willson et al. 1994), respondiendo de manera diferente cada una de las cuatro especies estudiadas, siendo P. tarnii unas de las más sensibles (Hansen \& Urban 1992), dado que presenta mayor 
ámbito de hogar en relación a las otras tres especies estudiadas para establecer su territorio. Para estas especies los lugares que presentan alteraciones en el medio ambiente conllevan a una baja presencia de individuos. Normalmente los rinocríptidos se rehúsan a atravesar áreas abiertas, siendo vulnerables a los efectos del aislamiento de los fragmentos (Sieving et al. 1996). La perturbación del bosque por plantaciones exóticas puede determinar la desaparición o alteración del sotobosque, fuente importante de material y cubierta de protección para los nidos de los tapaculos (Sieving et al. 2000).

Estas aves de los bosques templados de Sudamérica son vulnerables a la destrucción de su hábitat, debido a que la mayoría de los integrantes de esta familia son endémicas de esta región. Si el bosque nativo desaparece o se torna inhabitable, muchas de estas aves serán extirpadas o desaparecerán para siempre (Willson \& Armesto 2003). A ello se suma los riesgos de depredación de nidos (mayor en bosque fragmentado), los cuales podrían afectar la viabilidad de las poblaciones, hasta el punto de provocar extinciones locales (Vergara \& Simonetti 2003). Un depredador de nidos de rinocríptidos en la zona de estudios es el visón (Mustela vison, Schreber 1777), especie oportunista capaz de ingresar por los túneles realizados por estas aves y atacar los nidos (Willson et al. 2001), pudiendo depredar huevos e incluso polluelos (SAG 1999) (Guiñez com. pers.). Adicionalmente gatos y perros ferales contribuyen sin duda también a la mortalidad de aves, pero se carece de estudios específicos de su probable impacto en los bosques chilenos (Churcher \& Lawton 1987).

Cabe señalar también, que lo reducido del fragmento del área de estudio, puede incrementar el grado de competencia por el uso del hábitat, al igual que el grado de agresividad entre las especies de mayor tamaño corporal ( $P$. tarnii y $S$. rubecula), al ser más pequeñas las distancias entre los nidos de esta familia (Correa \& Figueroa 2003). La conectividad para muchas especies respecto de la mantención de los flujos genéticos es muy diferente para poblaciones de especies que están restringidas a vivir en el interior de los bosques (Willson 2004). Especies como el chucao ( $S$. rubecula), están extremadamente limitadas en su capacidad de ampliar o moverse entre parches de bosque y todos ellos requieren corredores de vegetación densa, virtualmente contiguos de bosque en el orden de mantener el flujo genético a través de la ecorregión. Los rinocríptidos presentan características que las hacen de especial relevancia para la conservación (UICN 2007), ya que son útiles para identificar hábitats bien conservados y definir prioridades de manejo y conservación (Lambeck 2002).

Glade (1993) establece en el Libro Rojo de los Vertebrados Terrestres de Chile de la Corporación Nacional Forestal (CONAF) que las cuatro especies de rinocríptidos presentes en el MNCÑ no se registran dentro de alguna categoría de conservación. Éste se basa en las categorías de UICN del año 1982, por lo que su vigencia hoy en día es sólo de carácter nacional dado por la Ley 19.300 (Ley de Bases del Medio 
Ambiente). El Servicio Agrícola y Ganadero (SAG 1998) en su Cartilla de caza, reglamento $\mathrm{N}^{\circ} 75$, artículo $3^{\circ}$ (criterios de protección de especies) de la Ley de caza 19,473 estipula que: P. tarnii, S. rubecula, E. paradoxa y S. magellanicus son especies catalogadas como beneficiosas para el sector silvoagropecuario (la cual se identifica con la letra "B"), fundamentalmente por su rol como controladores de plagas, en tanto que en su artículo $4^{\circ}$ (estados de conservación) de la Ley de caza, las especies anteriormente nombradas no se registran dentro de algún estado de conservación. Por su parte, la Cartilla de caza del SAG (2007) no hace mención alguna sobre la Familia Rhinocryptidae, ya que no son consideradas aves de caza, omitiéndolas con respecto a cualquier criterio o estado de conservación. Esta clasificación del SAG (2007) está basada en las categorías antiguas propuestas por UICN, las que hoy no se usan en el contexto mundial, puesto que las diferencias de criterios de clasificación son materia de debate constante y UICN actualmente se encuentra realizando una nueva revisión de su sistema. Pero el principal problema que tiene la clasificación realizada por el SAG es que se desarrolló en forma subjetiva, sin la necesaria utilización de información cuantitativa y actualizada de respaldo (Estades 2004), ante lo cual estas especies quedan desprotegidas.

Según la Unión Internacional para la Conservación de la Naturaleza (UICN 2004, 2007) las cuatro especies de la Familia Rhinocryptidae presentes en el MNCÑ se registran en la Lista Roja de las Aves Chilenas o Red List of Threatened Species Book (2007) como LC (Least Concern), es decir, especies de Preocupación Mínima. Las especies incluidas en esta categoría de conservación presentan según los datos (categorización) de la Lista Roja de UICN una cifra estimada de alcance mundial de ocurrencia de $120.000 \mathrm{~km}^{2}$ (E. paradoxa), $270.000 \mathrm{~km}^{2}$ (P. tarnii y $S$. rubecula) y $450.000 \mathrm{~km}^{2}$ (S. magellanicus), en las cuales las tendencias de las poblaciones a nivel mundial no han sido estimadas, y que seis de estas especies en el resto del mundo se registran dentro de algún criterio importante de conservación (UICN 2004, 2007). Sin embargo, se piensa que estas especies no han visto disminuida su población (disminución de más del 30\% en 10 años o tres generaciones), a pesar de no conocer la estimación real de las cuatro especies estudiadas, se hace necesaria la obtención de las tendencias poblacionales a nivel mundial.

Como se puede apreciar en el Cuadro 1 de acuerdo a los resultados obtenidos con el método Reca (Reca et al. 1994) se genera una discrepancia. Llama la atención que al utilizar este método el 50\% de la familia evaluada requieran Prioridad Máxima y Atención Especial de ser conservados. Esta técnica (Método Reca et al. 1994) también ha sido aplicada para evaluar el estado de conservación de distintos grupos de especies de Argentina: los tetrápodos del Parque Nacional Nahuel Huapi (Úbeda et al. 1994a, 1994b, Grigera et al. 1996), los tetrápodos de la región patagónica (Úbeda \& Grigera 1995), mamíferos de todo el país (Reca et al. 1996), reptiles y mamíferos del Parque Ischigualasto (Acosta \& Murúa 1998, 1999) y la totalidad de 
la herpetofauna Argentina (Lavilla et al. 2000, Úbeda \& Grigera 2003). También fue aplicado en Uruguay para evaluar las especies de anfibios y de algunos grupos de reptiles (Morales-Fagundes \& Carreira-Vidal 2000, Maneyro \& Langone 2001, Carreira-Vidal 2004) y en Chile se ha utilizado en anfibios (Díaz-Páez \& Ortiz 2003) y aves rapaces (Pincheira-Ulbrich et al. 2008), siendo considerada una herramienta útil de aplicación simple y económica, obteniendo resultados que permiten detectar amenazas actuales para cada taxa estudiado, presentándose esta identificación de suma utilidad para encaminar correctamente medidas de protección de las especies, revelando la importancia de la actual categorización de Reca con las preexistentes a través de métodos objetivos.

Si bien para aplicar el método Reca (Reca et al. 1994) no se requiere ser especialista si se dispone de la información sobre las especies, para la interpretación de los resultados y la asignación de las categorías, la discusión entre expertos es enriquecedora e incrementa la certeza en la categorización. Es evidente la necesidad de actualizar las categorizaciones periódicamente, con el objeto de que los administradores de la fauna tengan una herramienta útil que refleje lo más fielmente posible la situación de las especies en el país, situación dinámica y cambiante que resulta de las modificaciones de los hábitats, de las presiones directas sobre las poblaciones naturales y de la protección que sobre ellas se ejerce (Úbeda \& Grigera 2003).

Sin embargo, se recomienda ampliar y clarificar el contenido de algunas variables de la Metodología Reca (Reca et al. 1994) para efectos de evitar errores en la asignación de categorías.

Además de tener como limitante la carencia de datos básicos (conocimiento de la historia natural) para algunos grupos de especies. A pesar de ello, la metodología Reca (Reca et al. 1994) con los datos disponibles de la actualidad serviría como una herramienta para evitar que algunas especies queden desprotegidas por falta de priorización sobre éstas (Díaz-Páez et al. 2004), situación que acontece en la actualidad. No obstante, es necesario evaluar la complementación con otras metodologías de categorización, dependiendo del grupo taxonómico en estudio (Grigera \& Úbeda 2002). Además, para su efectiva aplicación es necesario integrar sus resultados con la normativa legal vigente y con acciones de monitoreo e investigación que permitan actualizar el conocimiento científico sobre la distribución y abundancia de las especies.

En Chile, tradicionalmente la protección de las aves ha tenido un sustento legal otorgado básicamente por la Ley de Caza del SAG e indirectamente por el SNASPE, además de otros cuerpos legales más recientes como la Ley de Bases del Medio Ambiente, pero no existe una ley específica sobre fauna silvestre que abarque los distintos aspectos relacionado con la conservación de las aves (Estades 2004), ya que los actuales leyes señalan la protección del medio ambiente, la preservación de la 
naturaleza y la conservación del patrimonio ambiental a grandes rasgos, sin especificar el grado de amenaza para cada taxón presente en Chile. En consecuencia, se hace urgente actualizar la legislación acorde a lo que realmente está sucediendo con las especies, mediante sistemas de clasificación actualizados y que demanden poco tiempo, ya que hoy en día la situación de la fauna a nivel mundial está cambiando constantemente, debido a la creciente intervención antrópica presente en sus hábitats.

El método Reca (Reca et al. 1994) es una técnica factible de ser aplicada, basada en criterios explícitos y cuantitativos que permite cuantificar el estado de conservación de especies de todas las clases de vertebrados tetrápodos, incluyendo las de distribución oceánica (Úbeda \& Grigera 1995), al mismo tiempo permite un tratamiento bastante efectivo de un importante grupo de taxa, adaptándose a las necesidades de dar respuestas rápidas a los tomadores de decisiones ambientales, los cuales deben tomar medidas actualizadas y prácticas. Por otro lado, para la ponderación de las variables se utilizan datos cuantitativos disponibles, permitiendo agregar o modificar variables para situaciones locales, cobrando especial importancia la abundancia local de especies, al hacerse más específica la real y actual situación (abundancia) de la Familia Rhinocryptidae en un lugar determinado para su conservación. Además, las categorías definidas por este método tienen una clara connotación jerárquica, permitiendo una mejor comprensión sobre como establecer prioridades, siendo las variables Potencial reproductivo (POTRE) y Amplitud trófica (AMTRO) muy importantes, ya que son indicadores de vulnerabilidad frente a disturbios. Así, este método se constituye en una herramienta útil para la gestión del recurso faunístico, hasta que se pueda incrementar el grado de conocimiento de las poblaciones y puedan utilizarse métodos más exactos (Grigera \& Úbeda 2000).

AGRADECIMIENTOS. Agradecemos los valiosos comentarios y revisiones de Esperanza Parada, que contribuyeron a enriquecer el trabajo. Pamela Sánchez y Basilio Guiñez por su disposición y ayuda prestada para el desarrollo del tema. Al administrador del Monumento Natural, Javier Vega por el apoyo brindado durante el trabajo de campo.

\section{LITERATURA CITADA}

Acosta, J. \& F. Murúa. 1998. Lista preliminar y estado de conservación de los reptiles del Parque Nacional Ishigualasto, San Juan, Argentina. Multequina, 7: 49-59.

Acosta, J. \& F. Murúa. 1999. Lista preliminar y estado de conservación de la mastofauna del Parque Natural Ischigualasto, San Juan, Argentina. Multequina, 8:121-129.

Araya, B., G. Millie \& M. Bernal. 1996. Guía de campo de las aves de Chile. Editorial Universitaria. Santiago, Chile. 404 pp.

Bibby, C., N. Burges \& D. Hili. 1993. Birds census techniques. Academic Press Limited. Second printing. London, Great Britain. 257 pp.

Carreira-Vidal, S. 2004. Estado de conservación de la fauna de Sauria y Amphisbaenidae (Reptilia, Squamata) de Uruguay. Cuadernos de Herpetología, 18:55-58. 
Churcher, P. \& J. Lawton. 1987. Predation by domestic cats in an English village. Journal of Zoology, 212: 439-455.

Centro de Información de Recursos Naturales (CIREN). 2002. Estudio agrológico. Descripciones de suelo, materiales y símbolos. Chile. $360 \mathrm{pp}$

Comisión Nacional del Medio Ambiente (CONAMA). 2002. Estrategia Regional de conservación y uso sustentable de la biodiversidad. Desarrollado en el marco de la elaboración de la Estrategia Nacional de conservación y uso sustentable de la biodiversidad. Región de la Araucanía, Chile. $170 \mathrm{pp}$.

Correa, A. \& J.A. Figueroa. 2003. Observations of aggressiveness and territoriality among species of Rhinocryptidae in a rain forest fragment in Southern Chile. Ornitologia Neotropical, 14: 121-125.

Di Castri, F. \& E. Hajek. 1976. Bioclimatología de Chile. Edic. Universidad Católica de Chile. Santiago, Chile. 131 pp.

Díaz-Páez, H. \& J. Ortiz. 2003. Evaluación del estado de conservación de los anfibios en Chile. Revista Chilena de Historia Natural, 76: 509-525.

Díaz-Páez, H., M. Vidal, P. Berríos, P. Bocaz, P. González, R. Moreno, W. Murillo, S. Rodríguez, R. Sánchez, R. Villagrán-Mella, J. Ortiz \& C. Úbeda. 2004. Revisión y análisis de los criterios y métodos para la categorización de las especies de flora y fauna en Chile. Boletín de la Sociedad de Biología de Concepción, 75: 73-89.

Estades, C. 2004. Estrategia Nacional para la Conservación de Aves 2004. Universidad de Chile. Programa interdisciplinario de estudios en biodiversidad. Santiago, Chile. 20 pp.

Feduccia, A. \& S. Olson. 1982. Morphological similarities between the Menurae and Rhinocryptidae, relict passefine birds of the Southern Hemisphere. Smithsonian Contributions to Zoology, 366:1-22.

Fjeldsâ, J. \& N. Krabbe. 1990. Birds of the high Andes. Copenhagen: Zool. Mus., Univ. of Copenhagen \& Svendborg: Apollo Books. 880 pp.

Gajardo, R. 1983. Sistema básico de clasificación de la vegetación nativa chilena. CONAF, FAO y Universidad de Chile. Facultad de Ciencias Agrarias, Veterinarias y Forestales. Santiago, Chile. 315 pp.

Glade, A. 1993. Libro rojo de los vertebrados terrestres de Chile. Actas del Simposio "Estado de Conservación de los Vertebrados Terrestres de Chile". Segunda edición. CONAF. Santiago, Chile. $67 \mathrm{pp}$.

Grigera, D. \& C. Úbeda. 2000. Una comparación de tres métodos para evaluar el estado de conservación de la fauna silvestre, mediante su aplicación a un conjunto de mamíferos patagónicos. Gestión Ambiental, 6: 55-71.

Grigera, D. \& C. Úbeda. 2002. Una revisión de los trabajos sobre categorizaciones y prioridades de conservación de los vertebrados de Argentina. Ecología Austral, 12: 163-174.

Grigera, D., C. Úbeda \& A. Reca. 1996. Estado de conservación de las aves del Parque y Reserva Nacional Nahuel Huapi. Hornero, 14: 1-13.

Hansen, A.J. \& D.I. Urban. 1992. Avian response to landscape pattern: the role of species life histories. Landscape Ecology, 7: 163-180.

Hauenstein, E., C. Ramírez \& M. Latsague. 1988. Evaluación Florística y Sinecológica del Monumento Natural Cerro Nielol (IX Región, Chile). Boletín Museo Regional Araucanía, Temuco, 3: 7-32.

Instituto Nacional de Estadísticas (INE). 1988. Compendio estadístico. Ministerio de Economía. Fomento y Reconstrucción. Imprenta INE. Santiago, Chile. 256 pp.

Köeppen, W. 1931. Die Klimate der Erde. Gundriss der Klimakunde. Berlín. 182 pp.

Lambeck, R. 2002. Focal species and restoration ecology. Conservation Biology, (16)2: 549-551.

Lavilla, E., E. Richard \& G. Scrocchi. 2000. Categorización de los anfibios y reptiles de la República Argentina. Asociación Herpetológica Argentina, San Miguel de Tucumán.97 pp. 
Maneyro, R. \& J.A. Langone. 2001. Categorización de los anfibios del Uruguay. Cuadernos de Herpetología, 15: 107-118.

Morales-Fagundes, S. \& S. Carreira-Vidal. 2000. Calificación del estado de conservación de la fauna de ofidios (Reptilia, Squamata, Serpentes) de Uruguay. Facena, 16: 45-51.

Pincheira-Ulbrich, J., J. Rodas-Trejo, V. Almanza \& J. Rau. 2008. Estado de conservación de las aves rapaces de Chile. Hornero, 23: 5-13.

Reca, A., C. Úbeda \& D. Grigera. 1994. Conservación de la fauna de tetrápodos. Un índice para su evaluación. Mastozoología Neotropical, 1: 17-28.

Reca, A., C. Úbeda \& D. Grigera. 1996. Prioridades de conservación de los mamíferos de la Argentina. Mastozoología Neotropical, 3: 87-117.

Rozzi, R., M. Martínez, M. Willson \& C. Sabag. 1996. Avifauna de los bosques templados de Sudamérica. En: Armesto J.J., C. Villagrán \& M.K. Arroyo (Eds). Ecología de los bosques nativos de Chile. 470 pp. Editorial Universitaria. Universidad de Chile. Primera Edición. Santiago, Chile.

Sarasola, M., B. Marqués \& V. Rush. 1999. Determinación de aptitud de hábitat para el pájaro carpintero patagónico (Campephilus magellanicus) como indicador de mantenimiento de la integridad de los sistemas bajo manejo forestal. Criterios e indicadores de manejo forestal sustentable. Biodiversidad III. Grupo Ecología Forestal. EEA -INTA. Bariloche, Argentina. 16 pp.

Servicio Agrícola y Ganadero (SAG). 1998. Ley 19.473. Cartilla de caza. Departamento de Recursos Naturales Renovables. Ministerio de Agricultura. Santiago, Chile. 84 pp.

Servicio Agrícola y Ganadero (SAG). 1999. El Visón (Mustela vison). Cartilla de caza. XI Región. Coyhaique, Chile. 12 pp.

Servicio Agrícola y Ganadero (SAG). 2007. Cartilla para cazadores. División de Protección de los Recursos Naturales Renovables. Ministerio de Agricultura. Santiago, Chile. 104 pp.

Sieving, K., M. Willson \& T.L. De Santo. 1996. Habitat barriers to movement of understory birds in fragmented south-temperate rainforest. The Auk, 113(4): 944-949.

Sieving, K., M. Willson \& T.L. De Santo. 2000. Defining corridor functions for endemic birds in fragmented south-temperate rainforest. Conservation Biology, 14: 1120-1132.

Tomaselli, R. 1981. The longitudinal zoning of vegetation in the southern sector of the Andes. Study trentini di scienze naturali, Acta Biológica, 58: 471-484.

Úbeda, C, \& D. Grigera. 1995. Recalificación del estado de conservación de la fauna silvestre argentina. Región Patagónica. Subsecretaria de Recursos Naturales y Ambiente Humano. Consejo asesor regional patagónico de la fauna silvestre, Buenos Aires. 95 pp.

Úbeda, C, \& D. Grigera. 2003. Análisis de la evaluación más reciente del estado de conservación de los anfibios y reptiles de Argentina. Gayana, 67(1): 97-113.

Úbeda, C, D. Grigera \& A. Reca. 1994a. Conservación de la fauna de tetrápodos. II. Estado de conservación de los mamíferos del Parque Nacional Nahuel Huapi. Mastozoología Neotropical, 1: 29-44.

Úbeda, C, D. Grigera \& A. Reca. 1994b. Estado de conservación de la herpetofauna del Parque y Reserva Nacional Nahuel Huapi. Cuadernos de Herpetología, 8: 155-163.

Unión Internacional para la Conservación de la Naturaleza (UICN). 2004. Lista roja de las aves chilenas. Gland, Suiza. 217 pp.

Unión Internacional para la Conservación de la Naturaleza (UICN). 2007. Lista roja de especies amenazadas. Gland, Suiza. 217 pp.

Vergara, P. \& J. Simonetti. 2003. Forest fragmentation and rhinocryptid nest predation in central Chile. Acta Oecologica, 24: 285-288.

Vuilleumier, F. 1985. Forests birds of Patagonia: ecological geography, speciation, endemism, and faunal history. Ornithological Monographs, 36: 255-305. 
Willson, M. 2004. Loss of habitat connectivity hinders pair formation and juvenile dispersal of Chucao tapaculos in Chilean rainforest. Condor 106: 166-171.

Willson, M. \& J.J. Armesto. 2003. Efectos de la fragmentación de bosques para las aves de los bosques australes chilenos. Ambiente y Desarrollo, (19): 3-4: 54-59.

Willson, M., T.L. De Santo, C. Sabag \& J.J. Armesto. 1994. Avian communities of fragmented South temperate rainforest in Chile. Conservation Biology, (8): 508-520.

Willson, M., J.L. Morrison, K. Sieving, T.L. De Santo, L. Santisteban \& I. Diaz. 2001. Patterns of predation risk and survival of bird nests in Chilean agricultural landscape. Conservation Biology, 15: 447-456. 


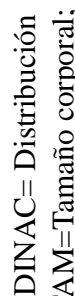

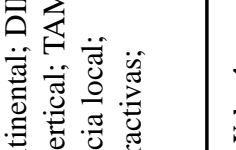

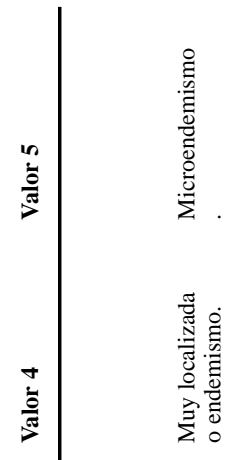

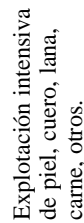

하응 중

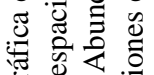

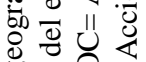

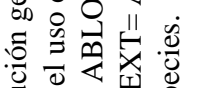

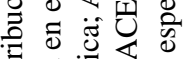

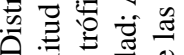

II 울 음

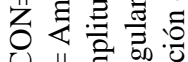

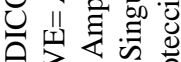

它出

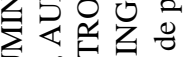

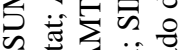

0 旸

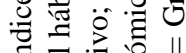

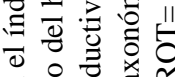

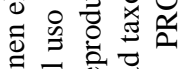

ฮ 뮹

형 휴 흥

를

पू

言尘焉

焉的

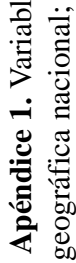

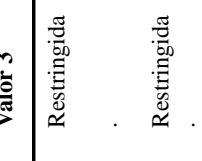

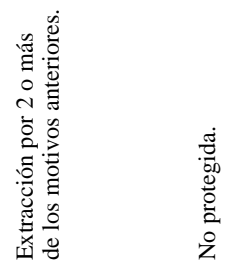

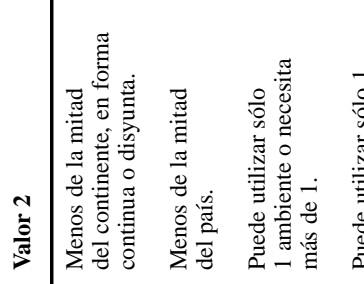

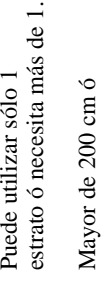

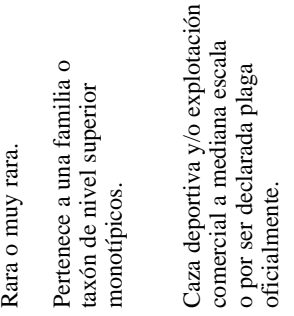



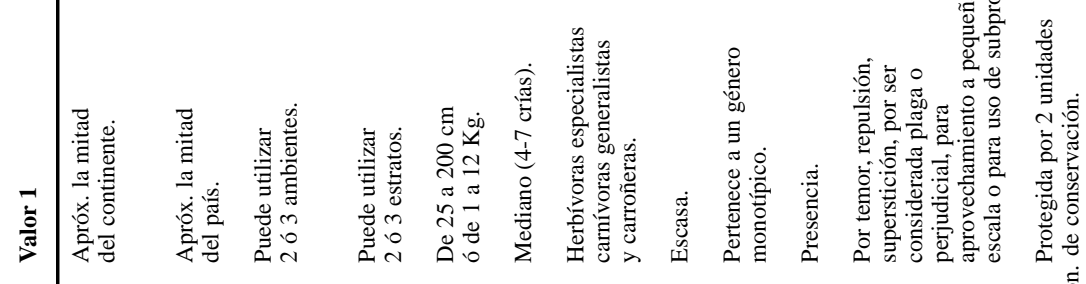

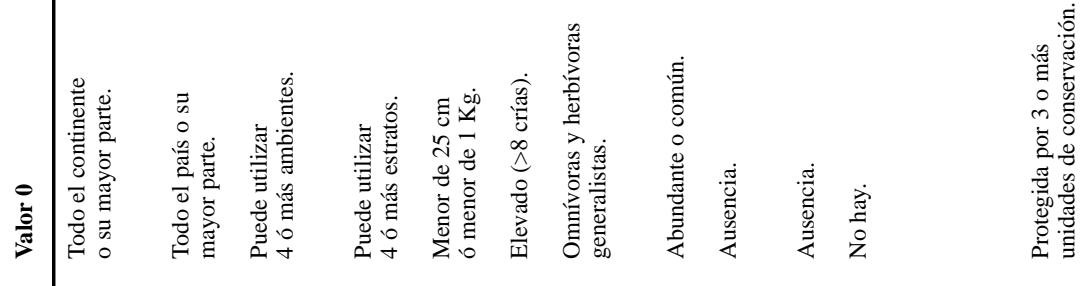

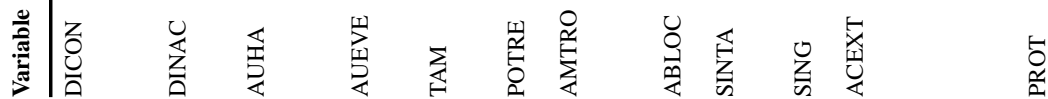




$$
\bar{I}
$$

